\title{
Fermion mass without symmetry breaking
}

\author{
Simon Catterall \\ Department of Physics, Syracuse University, \\ Syracuse, NY13244, U.S.A. \\ E-mail: smcatter@syr.edu
}

ABSTRACT: We examine a model of reduced staggered fermions in three dimensions interacting through an $\mathrm{SO}(4)$ invariant four fermion interaction. The model is similar to that considered in a recent paper by Ayyer and Chandrasekharan [1]. We present theoretical arguments and numerical evidence which support the idea that the system develops a mass gap for sufficiently strong four fermi coupling without producing a symmetry breaking fermion bilinear condensate. Massless and massive phases appear to be separated by a continuous phase transition.

KEYwORDs: Spontaneous Symmetry Breaking, Lattice Quantum Field Theory

ArXiv ePrint: 1510.04153 


\section{Contents}

1 Introduction 1

2 Lattice model and symmetries 2

3 Auxiliary field representation 5

4 Absence of bilinear condensates $\quad 7$

5 Phase structure $\quad 11$

6 Conclusions $\quad 15$

\section{Introduction}

In this paper we study a three dimensional lattice theory containing four fermion interactions invariant under an $\mathrm{SO}(4)$ symmetry. The structure of the model is motivated by recent work by Ayyar and Chandrasekharan [1] who construct a similar theory enjoying an $\mathrm{SU}(4)$ symmetry. The authors of that earlier work provide strong evidence that the system is capable of dynamically generating a fermion mass without producing a symmetry breaking bilinear condensate. Such a mechanism of mass generation is novel and potentially may find application in many areas of physics - for example it may offer alternative mechanisms to decouple mirror fermions in efforts to construct lattice chiral gauge theories - see the recent work in $[2,3]$ and the recent review [4] which provides a useful set of references to the earlier work in the lattice community.

The SO(4) invariant theory we study possesses practical advantages over the SU(4) model. The latter model possesses a sign problem and must be simulated using a worm algorithm. This limits the size of the system that can be studied. In contrast the $\mathrm{SO}(4)$ model we study in this paper does not suffer from a sign problem; after one replaces the four fermion interaction by suitable Yukawa terms, one can show that the Pfaffian that arises after integrating over the fermions is real, positive definite and hence can be simulated using a (rational) hybrid Monte Carlo algorithm.

In the next section we describe the lattice model and its symmetries and the phases expected at strong and weak four fermi coupling. The use of a reduced staggered fermion field ensures the absence of a single site mass term and so any $\mathrm{SO}(4)$ invariant mass term must be constructed from products of staggered fields which are neighbor to each other within an elementary (hyper)cube [5,6]. In fact the massless staggered quark action is invariant under a set of discrete shift symmetries which protect the theory against the generation of even these operators. Of course it is entirely possible that either the $\mathrm{SO}(4)$ or shift symmetries could break spontaneously at strong coupling and yield corresponding 
condensates and so much of our paper is devoted to providing numerical evidence to show that this does not occur. Instead we argue that the physics at strong coupling is instead driven by condensation of the symmetric four fermi operator appearing in the action. We show numerical evidence in favor of a phase transition to such a condensed phase for a critical value of the four fermi coupling. Furthermore, we show that the system develops a mass in this condensed phase. The use of reduced staggered fermions for constructing interesting lattice four fermion models was also emphasized in $[7,8]$. The latter work argued for the presence of a Higgs phase in a strongly coupled fermion theory although it has since been argued that this phase cannot survive the continuum limit [9].

Finally, since both these lattice symmetries are also present in four dimensions it is possible that this phase structure may also be present if the model is lifted to four dimensions.

\section{Lattice model and symmetries}

Consider a theory of reduced staggered fermions in three dimensions whose action contains a single site $\mathrm{SO}(4)$ invariant four fermion term. The action is given by

$$
S=\sum_{x} \sum_{\mu} \eta_{\mu}(x) \psi^{a}(x) \Delta_{\mu}^{a b} \psi^{b}(x)-\frac{1}{32} G^{2}\left(\sum_{x} \epsilon_{a b c d} \psi^{a}(x) \psi^{b}(x) \psi^{c}(x) \psi^{d}(x)\right)
$$

where $\Delta_{\mu}^{a b} \psi^{b}(x)=\frac{1}{2} \delta_{a b}\left(\psi^{b}(x+\hat{\mu})-\psi^{b}(x-\hat{\mu})\right)$ with $\hat{\mu}$ representing unit displacement in the lattice in the $\mu$-direction and $\eta_{\mu}(x)$ is the usual staggered fermion phase $\eta_{\mu}(x)=$ $(-1)^{\sum_{i=0}^{\mu-1} x_{i}}$. The (single component) reduced staggered fermions are taken to transform in the fundamental representation of $\mathrm{SO}(4)$ according to

$$
\psi(x) \rightarrow O \psi(x)
$$

with $O$ an arbitrary $\mathrm{SO}(4)$ rotation. If one employs periodic boundary conditions it is also invariant under the shift symmetry

$$
\psi(x) \rightarrow \xi_{\rho}(x) \psi(x+\hat{\rho})
$$

where the flavor phase $\xi_{\mu}(x)=(-1)^{\sum_{i=\mu+1}^{d-1} x_{i}}$. In four dimensions these shift symmetries can be thought of as a discrete remnant of continuum chiral symmetry [10].

These symmetries strongly constrain the possible bilinear terms that can occur in the theory. For example, a single site mass term of the form $\psi^{a}(x) \psi^{b}(x)$ breaks the $\mathrm{SO}(4)$ invariance but maintains the shift symmetry ${ }^{1}$ while $\mathrm{SO}(4)$ invariant bilinear terms constructed from products of staggered fields within the unit (hyper)cube generically break the shift symmetries $[5,6]$. The possible bilinear operators for a reduced staggered fermion

\footnotetext{
${ }^{1}$ The usual single site mass term that is possible for a full staggered field $\bar{\psi}^{a}(x) \psi^{a}(x)$ is invariant under both the $\mathrm{SO}(4)$ and shift symmetries but this term is absent for a reduced staggered field.
} 
that correspond to Dirac and Majorana masses in the continuum limit are listed below ${ }^{2}$

$$
\begin{aligned}
O_{1} & =\sum_{x, \mu} m_{\mu} \epsilon(x) \xi_{\mu}(x) \psi^{a}(x) S_{\mu} \psi^{a}(x) \\
O_{2}^{1} & =\sum_{x, \mu, \nu} m_{\mu \nu}^{1} \xi_{\mu}(x) \xi_{\nu}(x+\hat{\mu}) \psi^{a}(x) S_{\mu} S_{\nu} \psi^{a}(x) \\
O_{2}^{2} & =\sum_{x, \mu, \nu} m_{\mu \nu}^{2} \epsilon(x) \xi_{\mu}(x) \xi_{\nu}(x+\hat{\mu}) \psi^{a}(x) S_{\mu} S_{\nu} \psi^{a}(x) \\
O_{3} & =\sum_{x, \mu, \nu, \lambda} \hat{m}_{\mu \nu \lambda} \xi_{\mu}(x) \xi_{\nu}(x+\hat{\mu}) \xi_{\lambda}(x+\hat{\mu}+\hat{\nu}) \psi^{a}(x) S_{\mu} S_{\nu} S_{\lambda} \psi^{a}(x)
\end{aligned}
$$

where the site parity $\epsilon(x)=(-1)^{\sum_{i=0}^{d-1} x_{i}}$ and the coefficients $m_{\mu \nu}^{1}, m_{\mu \nu}^{2}, \hat{m}_{\mu \nu \lambda}=\hat{m} \epsilon_{\mu \nu \lambda}$ are totally antisymmetric in their indices. The symmetric translation operator $S_{\mu}$ acts on a lattice field according to

$$
S_{\mu} \psi(x)=\psi(x+\hat{\mu})+\psi(x-\hat{\mu})
$$

It is straightforward to show that these mass terms are all antisymmetric operators. As an example consider taking the transpose of the one link operator $O_{1}$.

$$
O_{1}^{T}=\sum_{x, \mu}-m_{\mu} \epsilon(x) \xi_{\mu}(x)[\psi(x+\hat{\mu}) \psi(x)+\psi(x-\hat{\mu}) \psi(x)]
$$

where we have have used the Grassmann nature of the fermions. Assuming periodic boundary conditions we can now shift the summation index $x$

$$
O_{1}^{T}=\sum_{x, \mu} m_{\mu} \epsilon(x) \xi_{\mu}(x) \psi(x)[\psi(x-\hat{\mu})+\psi(x+\hat{\mu})]=O_{1}
$$

This antisymmetric property is required of any bilinear operator acting on reduced staggered fermions. Let us now check the invariance properties of the one link operator under a shift symmetry associated with the direction $\rho$

$$
O_{1}^{(\rho)}=\sum_{x, \mu} m_{\mu} \epsilon(x) \xi_{\mu}(x) \xi_{\rho}(x) \xi_{\rho}(x+\hat{\mu}) \psi(x+\hat{\rho})[\psi(x+\hat{\mu}+\hat{\rho})+\psi(x-\hat{\mu}+\hat{\rho})]
$$

Again, assuming periodic boundary conditions we can shift the summation index $x$

$$
O_{1}^{(\rho)}=\sum_{x, \mu}-m_{\mu} \epsilon(x) \xi_{\mu}(x) \xi_{\mu}(\hat{\rho}) \xi_{\rho}(\hat{\mu}) \psi(x)[\psi(x+\hat{\mu})+\psi(x-\hat{\mu})]
$$

Using the result $\xi_{\mu}(\hat{\rho}) \xi_{\rho}(\hat{\mu})=\left(2 \delta_{\mu \rho}-1\right)$ we find

$$
O_{1}^{(\rho)}=O_{1}-2 m_{\rho} \epsilon(x) \xi_{\rho}(x) \psi(x)[\psi(x+\hat{\rho})+\psi(x-\hat{\rho})]
$$

Thus the expectation value of the second term on the r.h.s. of eqn 2.10 must hence vanish unless the corresponding shift symmetry is broken spontaneously. Similar conclusions

\footnotetext{
${ }^{2}$ We thank Jan Smit for useful conversations concerning the issue of reflection positivity with staggered fermions actions.
} 
can be obtained for the other multilink mass operators. The question of whether these symmetries break spontaneously, which is a prime focus of the current paper, requires a careful study of the finite volume system in the presence of a symmetry breaking external field. Spontaneous symmetry breaking is signaled by the presence of a non-zero bilinear condensate as the external source is sent to zero after the thermodynamic limit is taken. We will show numerical evidence later that this does not occur in this model.

Before turning to the auxiliary representation of the four fermi term and the subsequent numerical simulations we can first attempt to understand the behavior of the theory in the limits of both weak and strong coupling. At weak coupling one expects that the fermions are massless and there should be no bilinear condensate since the four fermi term is an irrelevant operator by power counting. In contrast the behavior of the system for large coupling can be deduced from a strong coupling expansion. The leading term corresponds to the static limit $G \rightarrow \infty$ in which the kinetic operator is dropped and the exponential of the four fermi term expanded in powers of $G$. In this limit the partition function is saturated by terms of the form

$$
Z \sim\left[G^{2} \int d \psi^{1}(x) d \psi^{2}(x) d \psi^{3}(x) d \psi^{4}(x) \psi^{1}(x) \psi^{2}(x) \psi^{3}(x) \psi^{4}(x)\right]^{V}
$$

corresponding to a single site four fermi condensate. Rescaling the fermion fields by $\sqrt{G}$ then facilitates a calculation of the the fermion propagator $G_{f}(x, y)=\left\langle\psi^{a}(x) \psi^{a}(y)\right\rangle$ by expanding the exponential of the kinetic operator each term of which carries a factor of $1 / G$.

$$
G_{f}(x, y) \sim \sum_{\text {paths } P_{(x \rightarrow y)}}\left(\frac{1}{G}\right)^{2 l(P)} \prod_{P}\left(M_{x x_{1}}\right)^{3}\left(M_{x_{1} x_{2}}\right)\left(M_{x_{2} x_{3}}\right)^{3}\left(M_{x_{3} x_{4}}\right) \ldots\left(M_{x_{n} y}\right)^{3}
$$

where $M_{x x+\mu}=\frac{1}{2} \eta_{\mu}(x)$ and $l(P)$ is the (odd) number of links along the path $P$. A similar calculation for the bosonic operator $G_{b}(x, y)=\left\langle\psi^{a}(x) \psi^{b}(x) \psi^{a}(y) \psi^{b}(y)\right\rangle$ yields an analogous expression

$$
G_{b}(x, y) \sim \sum_{\text {paths } P_{(x \rightarrow y)}}\left(\frac{1}{G}\right)^{2 l(P)} \prod_{P}\left(M_{x x_{1}}\right)^{2}\left(M_{x_{1} x_{2}}\right)^{2}\left(M_{x_{2} x_{3}}\right)^{3}\left(M_{x_{3} x_{4}}\right)^{2} \ldots\left(M_{x_{n} y}\right)^{2}
$$

In both cases the leading term corresponds to paths of minimal length and yields an exponential behavior for the correlation function with a mass $m \sim 2 \ln G$. Notice that the vanishing of the bosonic correlator for large separations implies that the corresponding fermion bilinear $<\psi^{a}(x) \psi^{b}(x)>$ is also zero in this limit.

Thus the strong coupling calculation indicates that for sufficiently large $G$ the system should realize a phase in which the fermions acquire a mass without breaking the $\mathrm{SO}(4)$ symmetry. At weak coupling we have argued that the symmetry is also unbroken but the fermions are massless. Clearly there has to be at least one phase transition separating these two phases and we will indeed provide evidence in favor of this later. But one can also conceive of a more complicated phase structure with multiple phase transitions between 
weak and strong coupling. Indeed the lattice theory we have been discussing is similar to Higgs-Yukawa models employing staggered or naive fermions that were studied earlier in [11-13] although those models differ crucially from the one considered in this paper since they employ a full staggered field and hence allow for symmetric bilinear mass terms. In particular while that earlier work provided evidence of symmetric phases at both weak and strong coupling a broken phase with a bilinear condensate was also observed at intermediate coupling. This intermediate phase was separated from its symmetric neighbors by first order phase transitions. We see no evidence of this intermediate phase in our model.

\section{Auxiliary field representation}

Following the usual Gross-Neveu strategy one can try to rewrite the theory in terms of Yukawa terms and auxiliary scalars. Specifically, if we define the antisymmetric fermion bilinear fields $\Sigma^{a b}=\psi^{a} \psi^{b}$ the four fermi term can be rewritten

$$
\frac{1}{16} G^{2} \sum_{x} \Sigma^{a b} \tilde{\Sigma}^{a b}
$$

where the dual fermion bilinear is given by $\tilde{\Sigma}^{a b}=\frac{1}{2} \epsilon_{a b c d} \Sigma^{c d}$. Furthermore if we introduce the (anti)self-dual fermion bilinear fields $\Sigma_{ \pm}^{a b}=\frac{1}{2}\left(\Sigma^{a b} \pm \frac{1}{2} \epsilon_{a b c d} \Sigma^{c d}\right)$ and exploit the Grassmann character of the underlying fermions this can be rewritten as

$$
\pm \frac{1}{8} G^{2}\left(\Sigma_{ \pm}^{a b}\right)^{2}
$$

Notice that $\Sigma_{ \pm}$transform in the fundamental representation under the corresponding $\mathrm{SO}_{ \pm}(3)$ subgroup of $\mathrm{SO}(4)$ (they are singlets under the other $\mathrm{SO}_{\mp}(3)$ symmetry)

$$
\mathrm{SO}(4)=\mathrm{SO}_{+}(3) \times \mathrm{SO}_{-}(3)
$$

The original action can then be generated by employing an auxiliary antisymmetric, selfdual boson field

$$
\phi_{+}^{a b}=\frac{1}{2}\left(\phi^{a b}+\frac{1}{2} \epsilon_{a b c d} \phi^{c d}\right)
$$

which transforms in the fundamental of $\mathrm{SO}_{+}(3)$ and is a singlet under $\mathrm{SO}_{-}(3){ }^{3}$

$$
\frac{G}{2} \phi_{+}^{a b} \Sigma_{+}^{a b}+\frac{1}{2}\left(\phi_{+}^{a b}\right)^{2}
$$

The primary advantage of writing the four fermion term using auxiliary fields is that then the fermions appear only quadratically in the action and so may be integrated out to yield a Pfaffian. In principle this allows one to simulate the model using the rational hybrid Monte Carlo algorithm [14]. However the latter algorithm requires that the Pfaffian be real, positive definite - the theory must not suffer from a sign problem. In fact we can show

\footnotetext{
${ }^{3}$ An similar action based on $\Sigma_{-}^{a b}$ can be used to construct the theory with $G^{2}<0$.
} 
that the self-dual property of the auxiliary field guarantees just this property. Consider the eigenvalue problem associated with the fermion operator

$$
\left(\eta_{\mu} \Delta_{\mu}+\frac{G}{2} \phi_{+}\right) \psi=\lambda \psi
$$

Since the operator is real and antisymmetric the eigenvalues $\lambda$ are pure imaginary and come in pairs $\lambda$ and $-\lambda$. One way to guarantee a positive Pfaffian is for the eigenvalues to also be doubly degenerate. It is easy to see that this is the case since the fermion operator is invariant under $\mathrm{SO}_{-}(3)$ transformations. Since $\psi$ transforms as the $\left(\frac{1}{2}, \frac{1}{2}\right)$ representation under $\mathrm{SO}(4)$ it will transform as a spinor under $\mathrm{SO}_{-}(3)$. Thus each level $\lambda$ will be doubly degenerate. This conclusion has been checked numerically and guarantees positivity of the Pfaffian.

If we now imagine integrating out the fermions we will generate an effective action for the auxiliary bosons $\phi_{a b}^{+}$of the form

$$
S_{\text {eff }}=-\frac{1}{2} \operatorname{Tr} \ln \left(\eta \cdot \Delta+\frac{G}{2} \phi_{+}\right)
$$

This can be expanded perturbatively in $G$ :

$$
S_{\text {eff }}=-\frac{1}{2} \operatorname{Tr} \ln (\eta \cdot \Delta)+\frac{1}{2} \sum_{n=1}^{\infty} \frac{(-1)^{n}}{n}\left(\frac{G}{2}\right)^{n} \operatorname{Tr}\left(M^{-1} \phi_{+}\right)^{n}
$$

where $M_{x y}^{a b}=\delta^{a b} \eta_{\mu}(x) \Delta_{x y}^{\mu}$. Rearranging this yields an expression for the $\mathrm{n}^{\text {th }}$ order vertex function of the auxiliary field.

$$
\Gamma^{n}\left(x_{1}, \ldots x_{n}\right) \sim \frac{(-1)^{n}}{2 n}\left(\frac{G}{2}\right)^{n} M_{x_{1} x_{2}}^{-1} M_{x_{2} x_{3}}^{-1} \ldots M_{x_{n} x_{1}}^{-1}
$$

The usual Gross-Neveu analysis of symmetry breaking proceeds from a consideration of the effective potential $V_{\text {eff }}\left(\phi_{+}\right)$which corresponds to the limit in which are the arguments of the $\phi_{+}$are set to a common value $x_{i} \rightarrow x$. But because of the antisymmetry of $M$ this onsite effective potential vanishes identically. Actually, one must be a little careful here; the free fermion operator has an exact zero mode which must be regulated in order to define $M^{-1}$ in a way compatible with the antisymmetry of $M$. This can be done using either a multilink mass term of the type discussed earlier or an antiperiodic boundary condition.

Of course a continuum effective potential could arise from any vertex function $\Gamma^{n}\left(x_{1}, \ldots x_{n}\right)$ when the arguments $x_{i}-x_{j}=O(a) i, j=1 \ldots n$ so one should be careful in drawing too strong a conclusion from this observation. Nevertheless it is at least a piece of evidence in support of the idea that this lattice model may evade the usual GrossNeveu symmetry breaking scenario. In general the propagator factors are real but not necessarily positive so that the structure of $\Gamma^{n}$ is quite complicated. There is one notable exception to this:

$$
\Gamma^{2}(x, y)=\frac{1}{2}\left(\delta_{x y}+\frac{G^{2}}{8}\left[M_{x y}^{-1}\right]^{2}\right)
$$

where we have added in the contribution from the classical action $\frac{1}{2}\left(\phi_{+}^{a b}\right)^{2}$. This corresponds to a positive definite nonlocal interaction mediated via the massless staggered fermions. 


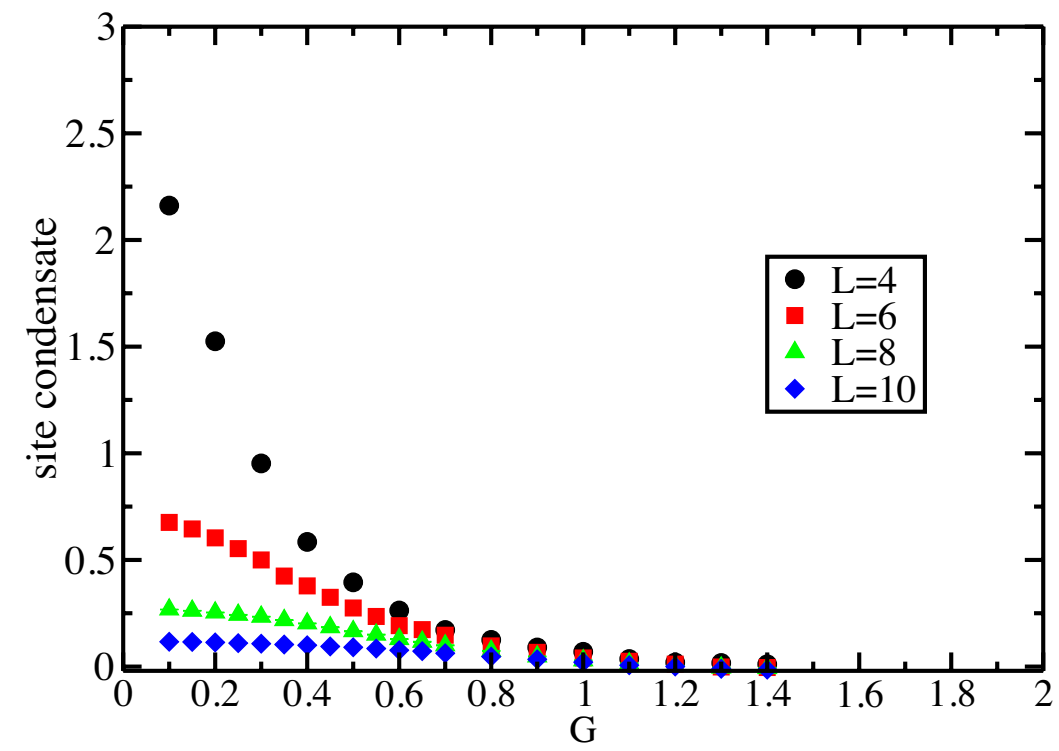

Figure 1. $<O_{0}>$ vs $G$ for $L=4,6,8,10$ and pbc.

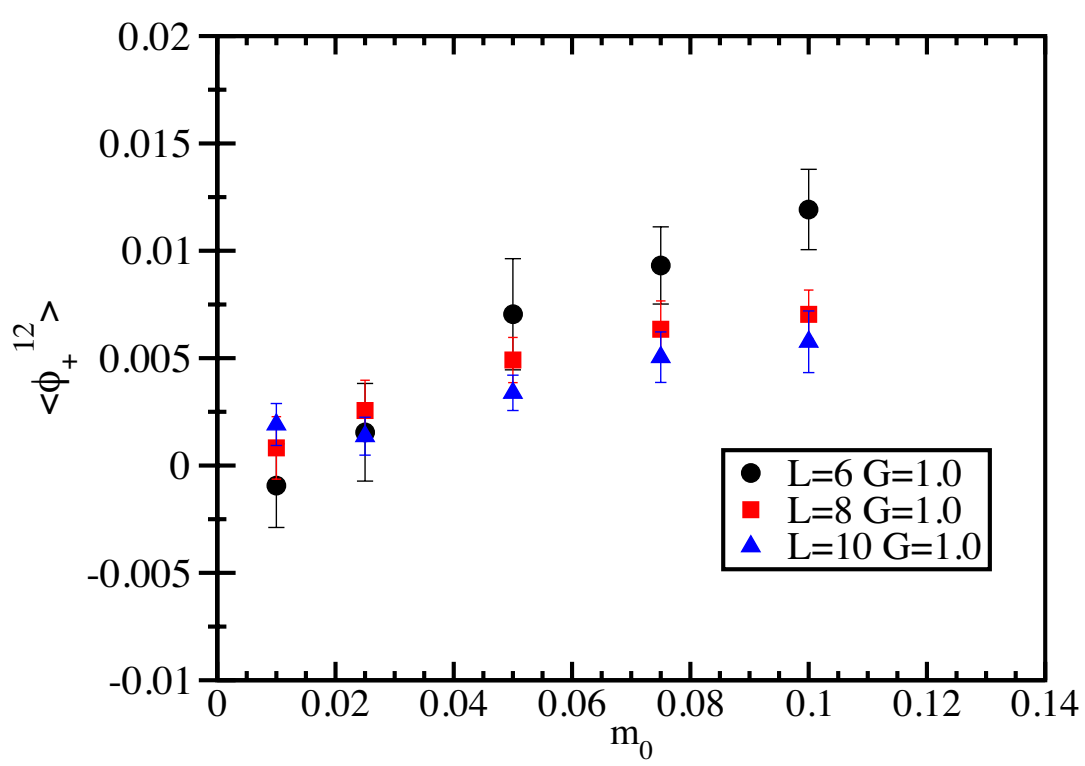

Figure 2. $<O_{0}>$ vs $m_{0}$ at $G=1.0$ for $L=6,8,10$ and pbc.

\section{Absence of bilinear condensates}

In the previous section we emphasized that the $\mathrm{SO}(4)$ and shift symmetries prevent the finite volume system from generating a non-zero vacuum expectation value for any of the fermion bilinears operators at finite volume. However these symmetries can in principle break spontaneously in the infinite volume limit - indeed this is what is expected to happen in QCD where strong gauge dynamics breaks chiral symmetries and yields a non-zero chiral condensate. However, in this section we will present evidence that this does not occur in this model. 
Consider first a possible site condensate of the form

$$
O_{0}^{a b}=\left[\psi(x)^{a} \psi^{b}(x)\right]_{+}
$$

where each index $a, b$ runs over the fundamental representation of $\mathrm{SO}(4)$ and the + subscript signifies as always that we are considering the selfdual component. Since we are interested in looking for symmetry breaking condensates we fix the values of these indices. For example figure 1. shows $\left\langle O_{0}^{12}\right\rangle$ (henceforth abbreviated to simply $O_{0}$ ) from simulations that utilize an external source which is non-zero only for $a=1$ and $b=2$. If such an operator were to develop a vacuum expectation value in the thermodynamic limit it would signal the breaking of $\mathrm{SO}(4) \rightarrow \mathrm{SO}_{-}(3) \times \mathrm{SO}_{+}(2)$ (again, note that $O_{0}^{a b}$ is a singlet under $\mathrm{SO}_{-}(3)$ ). To check for such a spontaneous breaking we have added an explicit mass term of this form with coupling $m_{0}=0.1$ to the lattice action and computed the expectation value of the single site condensate for a range of lattice volumes. Figure 1. shows the condensate as a function of the four fermi coupling. Since we employ periodic boundary conditions there is a large contribution to the expectation value from the near zero mode expected at small $G$. However as can be seen in the plot this contribution falls rapidly with increasing lattice volume. It is also clear that expectation value $\left\langle O_{0}\right\rangle$ is further suppressed as $G$ increases. This behavior points to the absence of spontaneous symmetry breaking. This conclusion can be cemented by examining the behavior of $O_{0}$ or equivalently $\phi_{+}^{12}$ at fixed $G$ and for several lattice volumes as the magnitude of the external source is varied. Figure 2. shows this quantity for fixed four fermi coupling $G=1.0$ as a function of the external source $m_{0}$. As expected the condensate approaches zero on any fixed volume as $m_{0} \rightarrow 0$ but more importantly the volume dependence is rather weak and indicates a condensate that is constant or decreasing with increasing volume. This is inconsistent with spontaneous symmetry breaking which would require that the measured condensate to increase with volume for sufficiently small $m_{0}$. Similar behavior is seen for all $G$. Thus the numerical results strongly suggest that the $\mathrm{SO}(4)$ symmetry does not break spontaneously. This is consistent with the results obtained in [1] for the SU(4) model.

Turning to the (hyper)cube mass operators we first analyze the one link operator $O_{1}$ defined in eq. (2.4). In our numerical work we consider mass parameters for the form $m_{\mu}=\left(m_{1}, m_{1}, m_{1}\right)$. Again we have examined the possibility that the shift symmetries break spontaneously by adding to the action a term of this form coupled to an external field $m_{1}$. Figure 3 . shows a plot of the measured link condensate versus $G$ for a range of lattice volumes and $m_{1}=0.1$. As before we employ periodic boundary conditions so that the only source of shift symmetry breaking lies in $m_{1}$. In a manner similar to the site operator we see a significant contribution to the link vev from the (near) fermion zero mode at small $G$ which is rapidly suppressed as the four fermi coupling increases. We have again checked that the limiting value of $\left\langle O_{1}\right\rangle$ approaches zero as $m_{1} \rightarrow 0$ in the large volume limit. Evidence in favor of this is presented in figure 4. which shows the link condensate at $G=1.0$ versus $m_{1}$ for three different lattice volumes. The volume dependence is rather small and there is no hint of a condensate growing with volume for small $m_{1}$ as would be expected if the condensate is to arise from spontaneous breaking of the shift symmetries. 


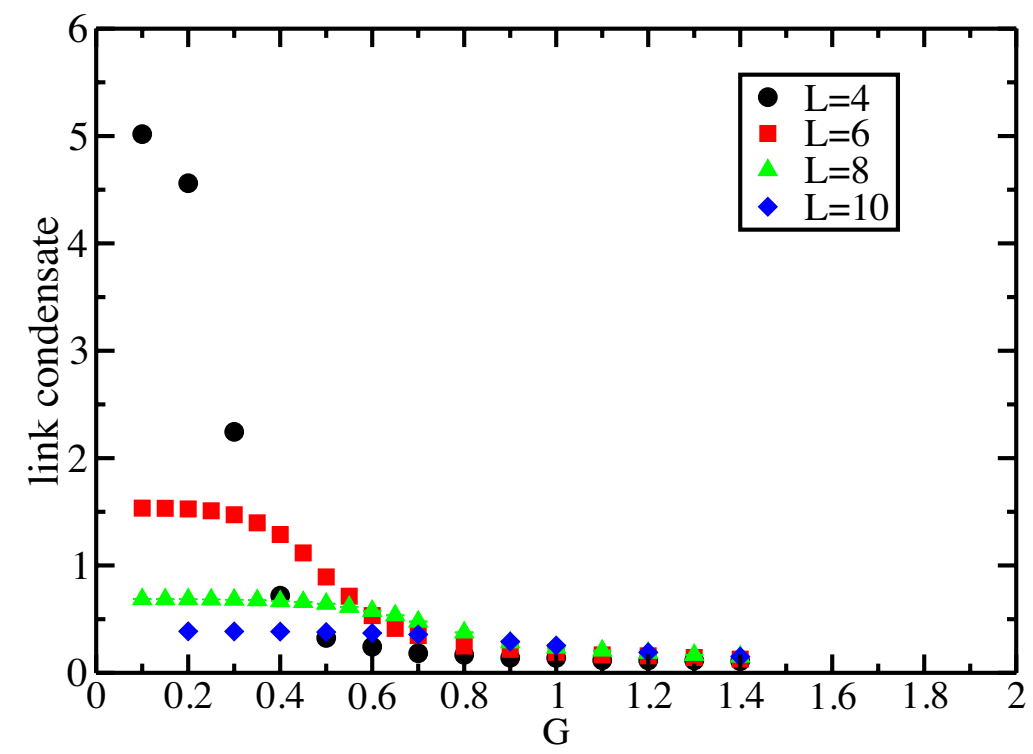

Figure 3. $<O_{1}>$ vs $G$ for $L=4,6,8,10$ and pbc.

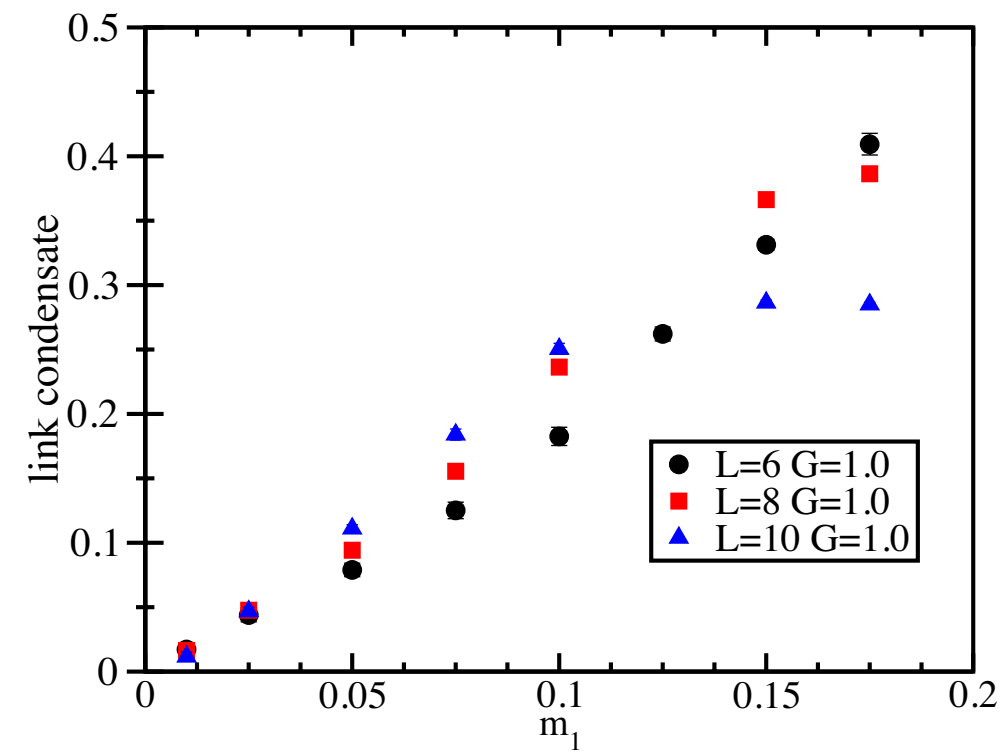

Figure 4. $<O_{1}>$ vs $m_{1}$ at $G=1.0$ for $L=6,8,10$ and pbc.

Indeed since the relevant dimensionless parameter governing a condensate $\Sigma$ is $x=m_{1} V \Sigma$ the slope of the curve in the case of spontaneous symmetry breaking should scale like $V$ as $m_{1} \rightarrow 0$ which is clearly not the case with our data.

We now turn to the examination of the two and three link mass operators described earlier. For these observables we show results obtained with an antiperiodic temporal boundary condition and $m_{0}=m_{1}=0$. Such a boundary condition explicitly breaks the shift symmetries and yields a fermion mass whose magnitude varies as $O(1 / L)$. It thus pro- 


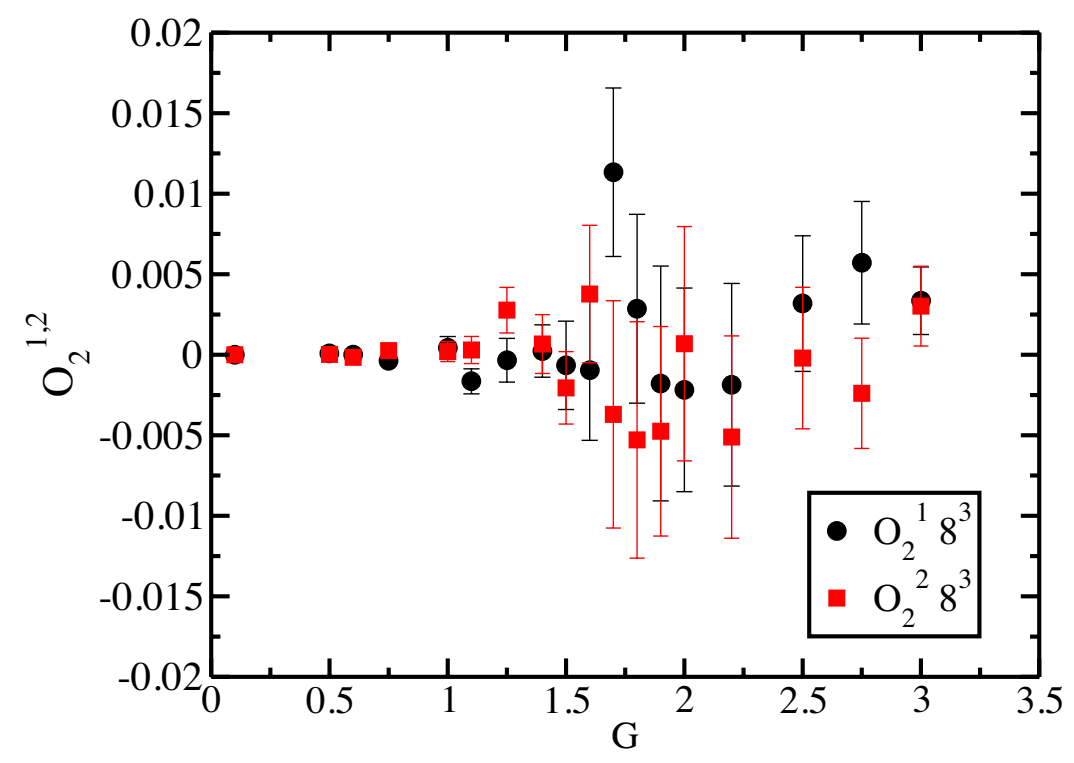

Figure 5. $<O_{2}^{1}>$ and $<O_{2}^{2}>$ vs $G$ for $L=8$ and temporal apbc.

vides a suitable way to look for spontaneous breaking of the shift symmetries by monitoring the vacuum expectation values of all (hyper)cube fermion bilinears simultaneously without introducing additional source terms. It has the added merit of automatically vanishing in the large volume limit. In the next section figure 10. presents results for the $G$ dependence of the one link operator which can be seen to be very similar to the previous results shown in figure 3. Using this boundary condition we have measured the vevs of both types of two link operator and also the three link operator. The two link terms are plotted in figure 5. for an $8^{3}$ lattice and show that these operators are statistically consistent with zero over the entire range of coupling $G$. The three link $O_{3}$ operator is shown in figure 6 . also for an $8^{3}$ lattice and again shows no sign of spontaneous symmetry breaking as the coupling $G$ is varied although an increase of the statistical errors is clearly visible in the vicinity of $G \sim 2$ which will turn out to coincide with a rapid increase in the vacuum expectation value of the four fermi operator. Evidence for spontaneous breaking of shift symmetry was presented in [15] for a gauge model in four dimensions. One of the order parameters used in that study involves the gauge field and hence has no analog in our system while the other $L_{\mu}$ can be written

$$
L_{\mu}=\sum_{x}(-1)^{x_{\mu}} \eta_{\mu}(x) \psi(x)[\psi(x+\hat{\mu})+\psi(x-\hat{\mu})]
$$

Using the result $\epsilon(x) \eta_{\mu}(x) \xi_{\mu}(x)=(-1)^{x_{\mu}}$ and summing over the index $\mu$ this can be shown to nothing more than the one link operator examined above and hence contributes nothing new to our analysis.

To summarize it appears that the shift and $\mathrm{SO}(4)$ symmetries do not break spontaneously for any value of the four fermi coupling. It is not surprising that the symmetries are intact at weak coupling but it is more surprising that they are not broken as the cou- 


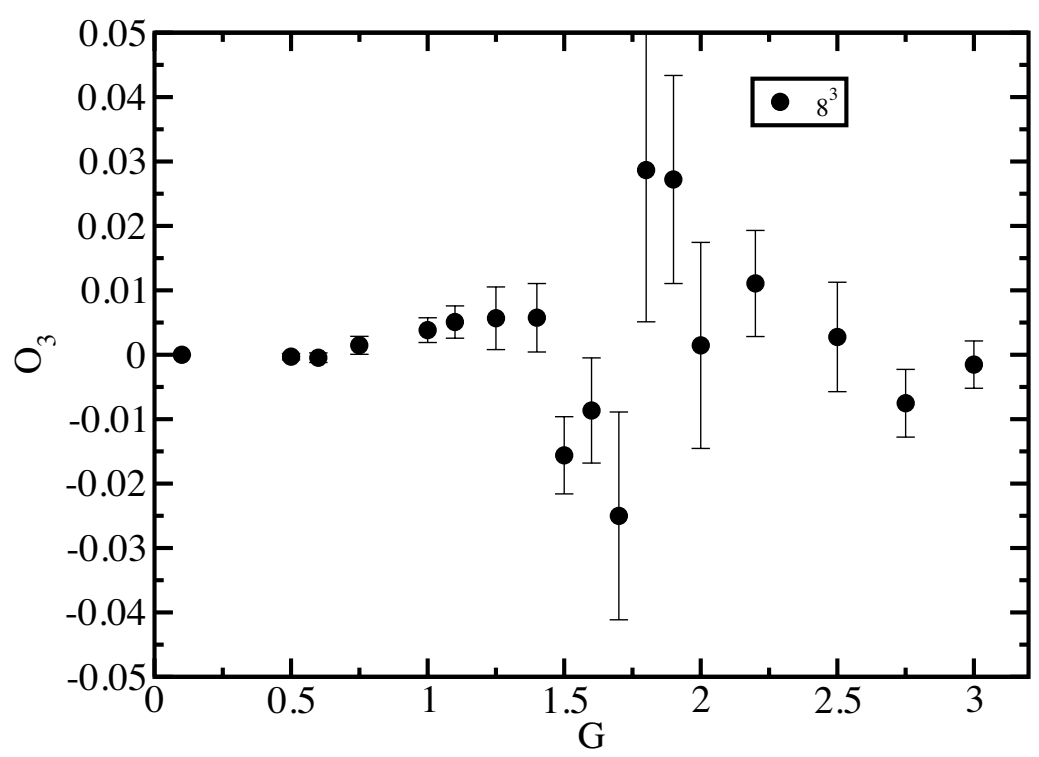

Figure 6. $<O_{3}>$ vs. $G$ with temporal apbc.

pling becomes stronger since fermionic systems can typically lower their energy by forming condensates. Actually, the existence of a strong coupled symmetric phase (PMS phase) had been observed before in a variety of lattice four fermion system - see the review [4]. However all these previous studies found an intermediate symmetry broken phase in which fermion bilinear condensates were formed. These seem to be absent in this model. Instead the system appears to evolve smoothly from a massless symmetric phase to the PMS phase with no intermediate symmetry breaking. In the next section we will provide evidence for this picture.

\section{$5 \quad$ Phase structure}

As discussed in the previous section we now set the external sources $m_{0}$ and $m_{1}$ to zero and instead use a thermal boundary condition to regulate the would be fermion zero mode in the weak coupling phase. To probe the phase structure of the theory we plot the expectation value of the four fermion operator $O_{4}=\frac{1}{4 !} \epsilon^{a b c d} \psi^{a} \psi^{b} \psi^{c} \psi^{d}$ as a function of the coupling $G{ }^{4}$

The expectation value of this operator is plotted in figure 7 . for two volumes $6^{3}$ and $8^{3}$ and shows a peak around $G \sim 2$. A related behavior is seen in the square of the auxiliary field $<\frac{1}{2} \phi_{+}^{2}>$ shown in figure 8. (actually we plot the quantity $<\phi_{+}^{2}>-\frac{3}{2}$ which vanishes at $G=0)$. The behavior of $\phi_{+}^{2}$ is consistent with the usual picture that the minimum of the effective potential for the auxiliary field moves away from the origin at strong coupling. What is different here is that while we observe a rapid increase of $\left\langle\phi_{+}^{2}\right\rangle$ for some $G$ we find good evidence that $\left\langle\phi_{+}\right\rangle=0$ for all $G$. This is made clear in figure 9 . which plots the expectation value of the single site fermion bilinear (the equivalent plot for $\phi_{+}$itself is

\footnotetext{
${ }^{4}$ Each ensemble used for the phase structure analysis at fixed $G$ and $L$ consists of 1000 configurations each separated by 10 HMC trajectories.
} 


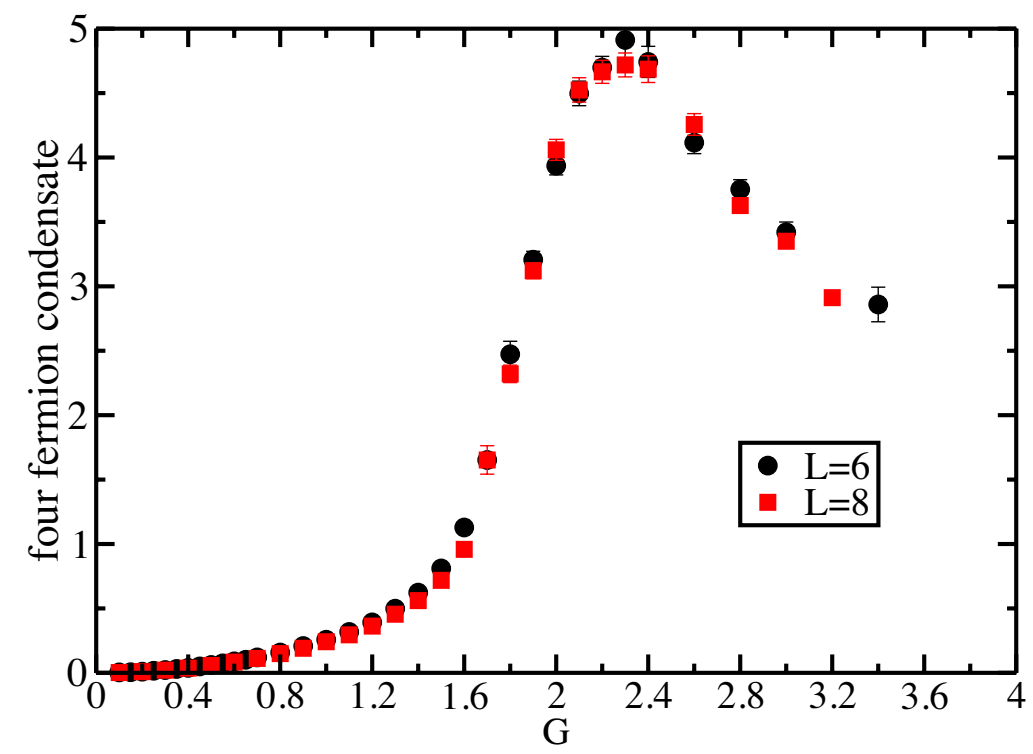

Figure 7. $\frac{1}{4 !}<\epsilon^{a b c d} \psi^{a} \psi^{b} \psi^{c} \psi^{d}>$ vs $G$ with temporal apbc.

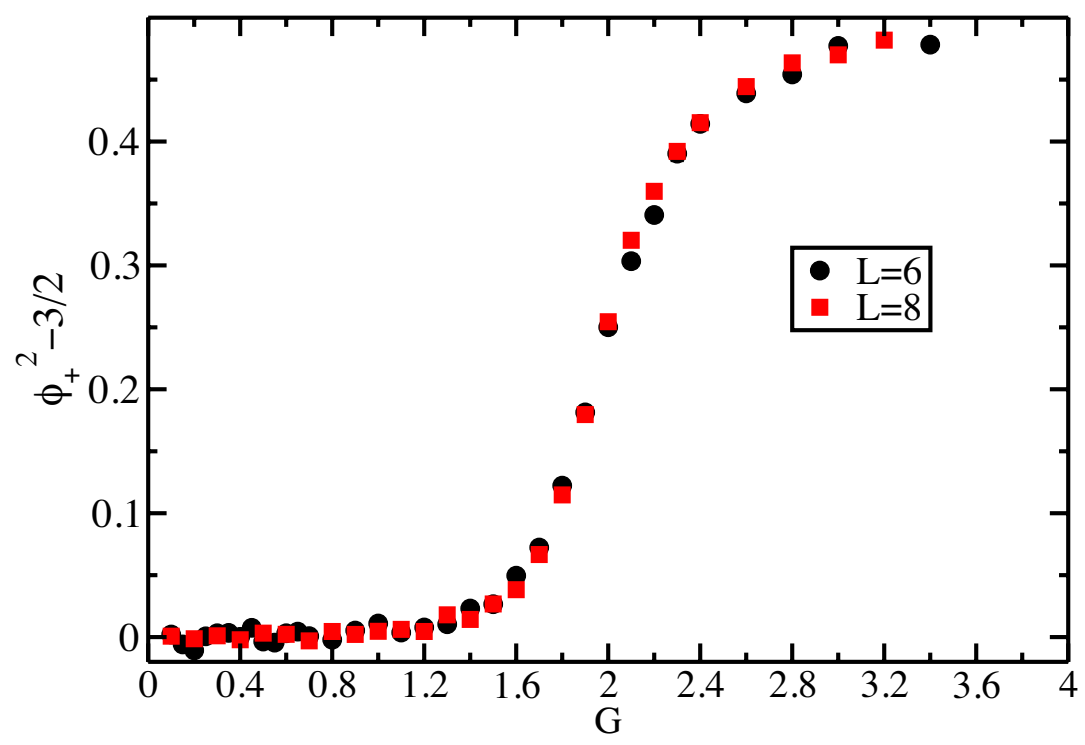

Figure 8. $<\phi_{+}^{2}>$ vs $G$ with temporal apbc.

very similar). This behavior is quite different from that seen in the standard Gross-Neveu scenario and indicates that the mechanism driving any phase transition in the model is quite different.

Notice that the thermal boundary conditions do force a non-zero value for the link operator in figure 10. at weak coupling but this is suppressed as the four fermi condensate is formed. Furthermore, notice that the value of this link condensate is approximately constant until the phase transition associated with the formation of the four fermion con- 


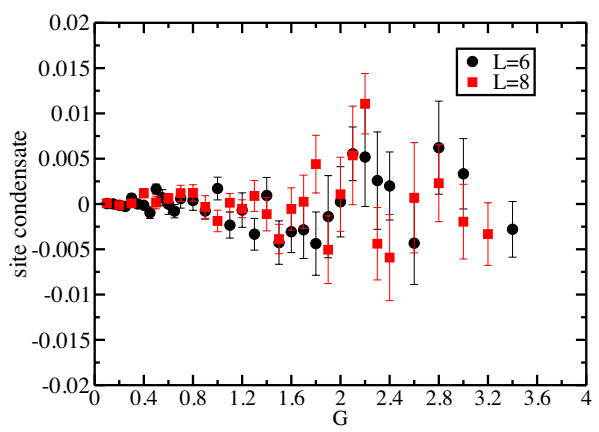

Figure 9. $O_{0}$ vs. $G$ with temporal apbc.

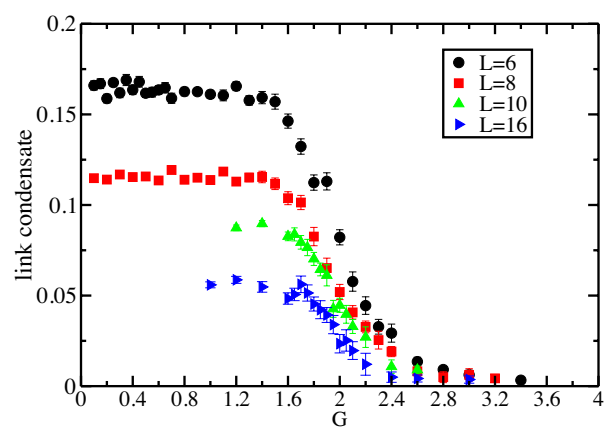

Figure 10. $O_{1}$ vs. $G$ with temporal apbc.

densate is reached - there is no sign of an intermediate broken phase which would be associated with an enhanced bilinear condensate. The non-zero value of the link operator at weak coupling is best thought of as arising from an effective link mass term originating in the thermal boundary conditions. Indeed, the plot makes it clear that the value of the link operator at weak coupling goes to zero like $1 / L$ as the lattice size increases. Thus in the thermodynamic limit our numerical work points to a vanishing link condensate for all values of the coupling $G$.

Next we turn to an observable which shows clearly the presence of a single phase transition at strong coupling. This is the susceptibility $\chi$ given by

$$
\chi=\frac{1}{L^{3}} \sum_{a, b, x \neq y}\left\langle\psi^{a}(x) \psi^{b}(x) \psi^{a}(y) \psi^{b}(y)\right\rangle
$$

This is plotted in figure 11. for lattice volume $L=6^{3}, 8^{3}, 10^{3}, 12^{3}$ and shows a single growing peak around $G_{c} \sim 1.9$ separating the weak and strong coupling regimes. The current lattices are too small for the reliable extraction of critical exponents using finite size scaling and we leave that for future work. Nevertheless figure 11. makes it clear that the peak height is growing much more slowly than the volume of the lattice as would be expected for a system undergoing a first order phase transition. Hence our current results favor a continuous phase transition. Finally we turn to the question of the mass of the fermions. Figure 12. show a plot of the following spatially averaged four fermion correlation function computed on a $8^{2} \times 16$ lattice

$$
\begin{aligned}
G(t) & =\sum_{x, y, a, b}\left\langle\psi^{a}(x, t) \psi^{b}(x, t) \psi^{a}(y, 0) \psi^{b}(y, 0)\right\rangle \\
& =\sum_{x, y, a, b}\left\langle\psi^{a}(x, t) \psi^{b}(y, 0)\right\rangle\left\langle\psi^{b}(x, t) \psi^{a}(y, 0)\right\rangle-\left\langle\psi^{a}(x, t) \psi^{a}(y, 0)\right\rangle^{2}
\end{aligned}
$$

This quantity when integrated over Euclidean time yields the susceptibility considered earlier. We show this correlator in three regimes: weak coupling $G=1.65$, strong coupling $G=3.0$ and close to the phase transition $G=1.9$. As expected the measured mass is small at weak coupling but becomes large $O(1)$ at strong four fermi coupling. Except for perhaps the $G=1.9$ data the linearity of the log plots is consistent with the correlators 


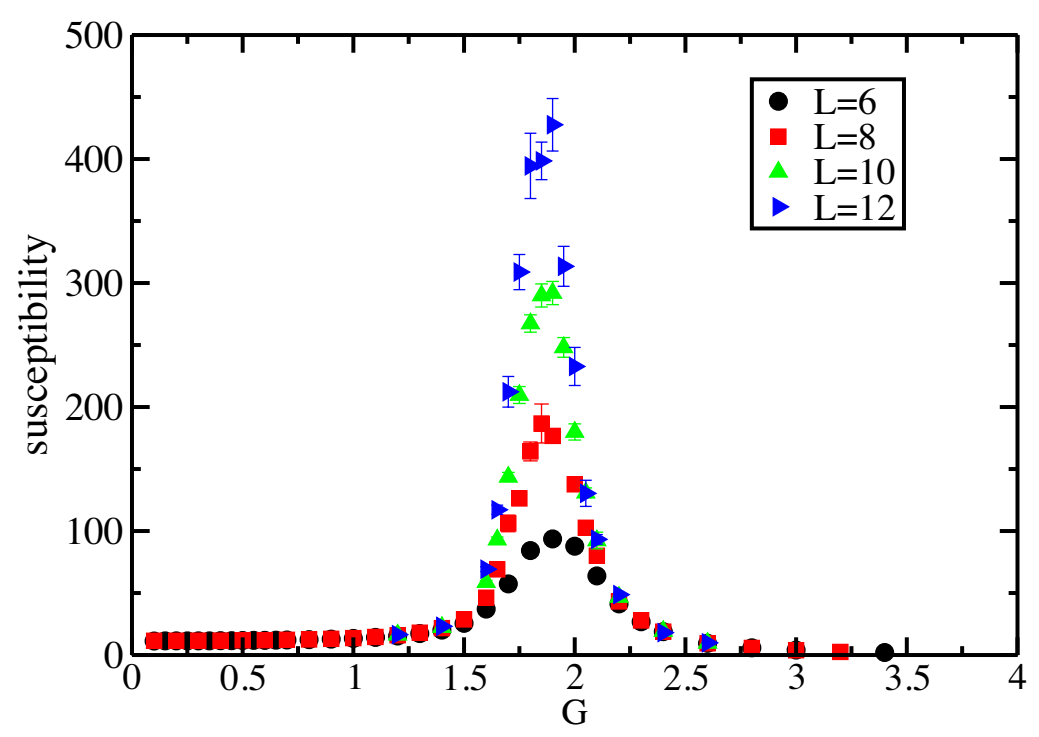

Figure 11. $\chi$ vs $G$ for $L=6,8,10,12$ for temporal apbc.

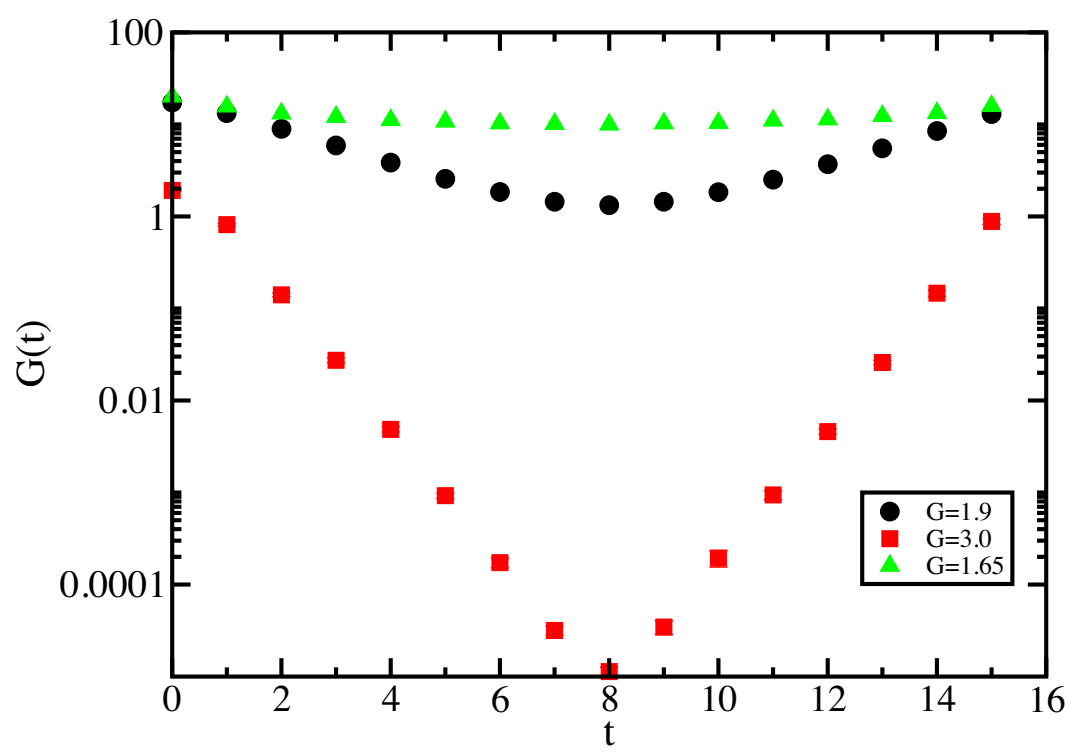

Figure 12. $G(t)$ vs $t$ for $G=1.65, G=1.9$ and $G=3.0$.

being dominated by a single state for large time separations. Using this information we have fitted the correlation function to a simple cosh form to extract an estimate of the mass of the $\psi^{a} \psi^{b}$ state - see the plot in figure 13. Notice that the small mass visible in the weak coupling phase is consistent with the magnitude of the measured one link condensate induced via the use of the temporal antiperiodic boundary condition. We hence expect the mass to vanish in the thermodynamic limit.

To summarize our simulations indicate that the model exhibits a two phase structure; at weak coupling the fermions are massless while at strong coupling they acquire a dynam- 


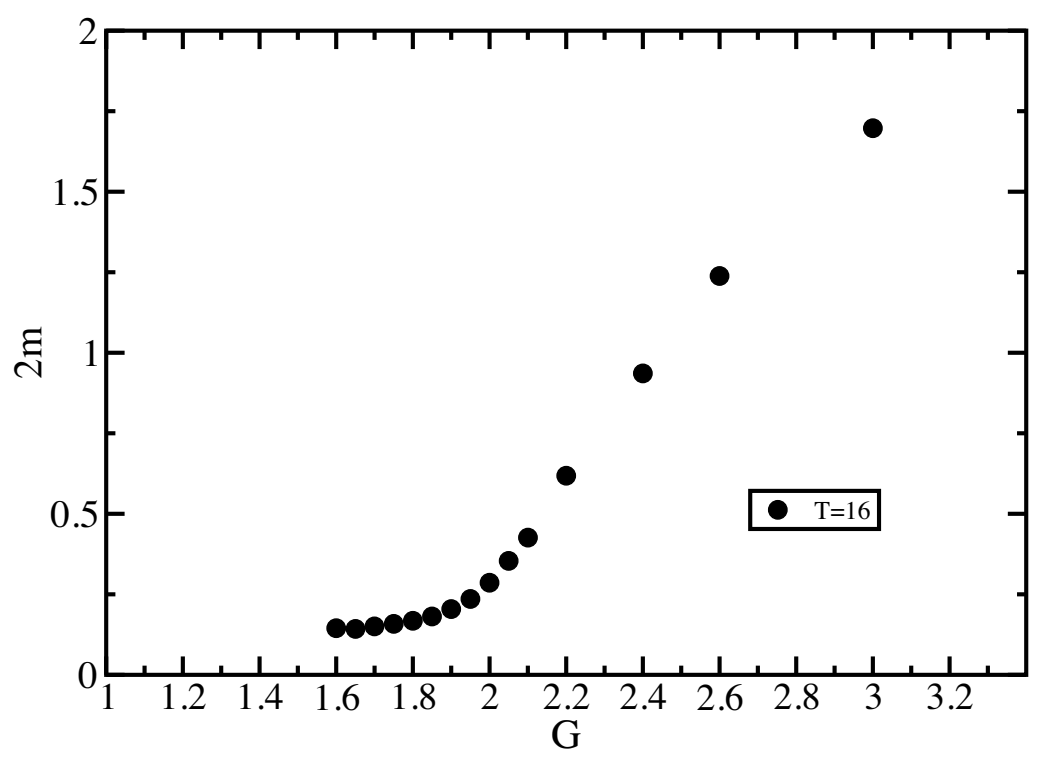

Figure 13. Fermion mass vs $G$ for temporal apbc.

ical mass and the system develops a four fermion condensate. A single continuous phase transition separates these two regimes but unlike the usual Gross-Neveu scenario there is no local order parameter - all fermion bilinears vanish at all values of the four fermi coupling and the mass generation is not associated with a spontaneous breaking of lattice symmetries.

\section{Conclusions}

In this paper we have studied a three dimensional lattice theory of reduced staggered fermions coupled through a $\mathrm{SO}(4)$ invariant four fermion interaction. We have put forward theoretical arguments based on exact lattice symmetries together with the results of numerical simulations to make the case that bilinear fermion condensates do not form in the theory for any value of the four fermi coupling $G$. This contrasts sharply from previous lattice studies of four fermion theories - see the reviews $[4,16]$ and references therein. At weak coupling we observe a conventional paramagnetic (PMW) phase while for large values of the coupling a strongly coupled (PMS) phase is observed characterized by a symmetric four fermion condensate. Both of these phases had been seen before - see $[11,17]$. The existence of the PMS phase and the resulting gapped spectrum is likely a robust feature of many strong coupling systems. The new aspect of both our work and that of [1] is that unlike the earlier studies we find no evidence of a conventional symmetry broken phase at intermediate coupling - the weak and strong coupling regimes appear to be separated by a single phase transition. Furthermore this transition appears to be continuous in contrast to the first order transitions separating the PMS and PMW phases from the intermediate broken phase. This latter feature is potentially very important; it suggests that it might be possible to construct a gapped continuum theory close to the phase transition. This should 
be contrasted with the older work where the masses generated in the PMS are necessarily cut-off scale. If it is indeed possible to build a continuum theory with a finite mass gap close to this putative continuous phase transition it would constitute a new mechanism for mass generation which is not tied to the formation of a symmetry breaking condensate.

The essential ingredients that appear to be important in constructing strongly coupled lattice fermion models that generate a mass gap without symmetry breaking are rather restrictive and include

- The model must not admit symmetric fermion bilinears. If these are allowed the system will most likely choose to condense such operators.

- The exact symmetries, the dimension of the fermion representation and the structure of the multifermion operator appearing in the action are intimately related; in the case of a four fermion term this seems to require (reduced staggered) fermions appearing in the fundamental representation of either an $\mathrm{SU}(4)$ or $\mathrm{SO}(4)$ symmetry group.

- The dynamics should be such as to render a symmetric four fermion condensate energetically favorable in comparison to a symmetry breaking bilinear condensate. While it is plausible that the ground state of a strongly interacting system breaks the minimal number of exact symmetries this is ultimately a dynamical and nonperturbative question.

The problem of using quartic interactions to gap out fermions without breaking symmetries has also received a lot of attention in recent years from the condensed matter community in the context of topological insulators and superconductors. It is intriguing that the counting of fermions required in those Hamiltonian constructions matches closely with the counting of (reduced) staggered quarks in three and four dimensions [2, 3, 18, 19]. The staggered quark symmetries seem to play a similar role to time reversal symmetry in the CMT constructions. Indeed, It is possible that the model discussed here is related to that discussed in [20].

Clearly there is much work to be done in elucidating the nature of the phase diagram and the precise connection, if its exists, to these condensed matter systems. We regard the current simulations as a "proof of principle" and to establish definitive results and reliable estimates for critical exponents one will need to simulate much larger lattices. We hope to report on such results in the near future. The question of the continuum limit is also of primary importance. Since $G$ is an irrelevant operator it should flow to zero in the IR and the physics of the massless phase will be dominated by the trivial fixed point at $G=0$. Any fixed point at $G=\infty$ would correspond to zero correlation length and have no continuum counterpart. The interesting question is whether a new continuum limit can be obtained by tuning $G$ towards $G_{c}$ assuming the transition is indeed continuous. The nature of this continuum theory is course very interesting; given the antisynmmetry of the fermion operator it is natural to consider constructing a continuum theory in which the reduced staggered field gives rise to four Majorana fermions. However, a simple transcription of the lattice action will not work - the required four fermion term vanishes identically

$$
\epsilon_{a b c d} \psi_{\alpha}^{a} C_{\alpha \beta} \psi_{\beta}^{b} \psi_{\gamma}^{c} C_{\gamma \delta} \psi_{\delta}^{d}=0
$$


Perhaps the most pressing question though, is whether the phase structure we have seen in three dimensions survives to four dimensions. The symmetries prohibiting bilinear terms remain the same so it is an open question. Whether any phase transition between weak and strong coupling remains continuous is another question; the usual universality arguments would place this model in the same class as Higgs-Yukawa systems [21] and so one would expect that any phase transition in four dimensions should either be first order or have mean field critical exponents. Any evidence to the contrary would be very exciting!

\section{Acknowledgments}

SMC is supported in part by DOE grant DE-SC0009998 and would like thank Poul Damgaard, Anna Hasenfratz, David Schaich, Uwe Wiese, Rohana Wijewardhana, Cenke $\mathrm{Xu}$ and especially Shailesh Chandrasekharan for useful discussions. SMC would also like to acknowledge the Aspen Center for Physics (NSF grant PHY-1066293) and the Kavli Institute for Theoretical Physics (NSF PHY11-25915) for support during the completion of this work. Numerical simulations were performed at Fermilab using USQCD resources.

Open Access. This article is distributed under the terms of the Creative Commons Attribution License (CC-BY 4.0), which permits any use, distribution and reproduction in any medium, provided the original author(s) and source are credited.

\section{References}

[1] V. Ayyar and S. Chandrasekharan, Massive fermions without fermion bilinear condensates, Phys. Rev. D 91 (2015) 065035 [arXiv:1410.6474] [INSPIRE].

[2] Y. BenTov and A. Zee, The origin of families and $\mathrm{SO}(18)$ grand unification, arXiv: 1505.04312 [INSPIRE].

[3] Y.-Z. You and C. Xu, Interacting topological insulator and emergent grand unified theory, Phys. Rev. B 91 (2015) 125147 [arXiv:1412.4784] [INSPIRE].

[4] E. Poppitz and Y. Shang, Chiral lattice gauge theories via mirror-fermion decoupling: a mission (im)possible?, Int. J. Mod. Phys. A 25 (2010) 2761.

[5] C. van den Doel and J. Smit, Dynamical symmetry breaking in two flavor $\mathrm{SU}(N)$ and $\mathrm{SO}(N)$ lattice gauge theories, Nucl. Phys. B 228 (1983) 122 [InSPIRE].

[6] M.F.L. Golterman and J. Smit, Selfenergy and flavor interpretation of staggered fermions, Nucl. Phys. B 245 (1984) 61 [inSPIRE].

[7] S. Catterall and A. Veernala, Four fermion interactions in non-abelian gauge theory, Phys. Rev. D 87 (2013) 114507 [arXiv:1303.6187] [InSPIRE].

[8] S. Catterall and A. Veernala, Dynamical gauge symmetry breaking in strongly coupled lattice theories, Phys. Rev. D 88 (2013) 114510 [arXiv:1306.5668] [InSPIRE].

[9] M. Golterman and Y. Shamir, Vacuum alignment and lattice artifacts: Wilson fermions, Phys. Rev. D 89 (2014) 054501 [arXiv: 1401. 0356] [InSPIRE].

[10] W. Bock, J. Smit and J.C. Vink, Fermion Higgs model with reduced staggered fermions, Phys. Lett. B 291 (1992) 297 [hep-lat/9206008] [INSPIRE]. 
[11] A. Hasenfratz and T. Neuhaus, Nonperturbative study of the strongly coupled scalar fermion model, Phys. Lett. B 220 (1989) 435 [INSPIRE].

[12] I.-H. Lee, J. Shigemitsu and R.E. Shrock, Lattice study of a Yukawa theory with a real scalar field, Nucl. Phys. B 330 (1990) 225 [inSPIRE].

[13] D. Stephenson and A. Thornton, Nonperturbative Yukawa couplings, Phys. Lett. B 212 (1988) 479 [INSPIRE].

[14] M.A. Clark, A.D. Kennedy and Z. Sroczynski, Exact $2+1$ flavour RHMC simulations, Nucl. Phys. Proc. Suppl. 140 (2005) 835 [hep-lat/0409133] [INSPIRE].

[15] A. Cheng, A. Hasenfratz and D. Schaich, Novel phase in SU(3) lattice gauge theory with 12 light fermions, Phys. Rev. D 85 (2012) 094509 [arXiv:1111.2317] [InSPIRE].

[16] S. Hands, Fixed point four Fermi theories, in the proceedings of APCTP-ICTP Joint International Conference on Recent developments in nonperturbative quantum field theory, May 26-30, Seoul, Korea (1997).

[17] M.F.L. Golterman, D.N. Petcher and E. Rivas, Absence of chiral fermions in the Eichten-Preskill model, Nucl. Phys. B 395 (1993) 596 [hep-lat/9206010] [INSPIRE].

[18] L. Fidkowski and A. Kitaev, The effects of interactions on the topological classification of free fermion systems, arXiv:0904.2197 [INSPIRE].

[19] T. Morimoto, A. Furusaki and C. Mudry, Breakdown of the topological classification $\mathbb{Z}$ for gapped phases of noninteracting fermions by quartic interactions, Phys. Rev. B 92 (2015) 125104 [arXiv: 1505.06341] [INSPIRE].

[20] K. Slagle, Y.-Z. You and C. Xu, Exotic quantum phase transitions of strongly interacting topological insulators, Phys. Rev. B 91 (2015) 115121 [arXiv:1409.7401] [INSPIRE].

[21] A. Hasenfratz, P. Hasenfratz, K. Jansen, J. Kuti and Y. Shen, The equivalence of the top quark condensate and the elementary Higgs field, Nucl. Phys. B 365 (1991) 79 [InSPIRE]. 